\title{
Uso ético da biodiversidade brasileira: necessidade e oportunidade
}

\author{
C.S. Funari*, V.O. Ferro \\ Departamento de Farmácia, Faculdade de Ciências Farmacêuticas, Universidade de São Paulo, \\ Av. Lineu Prestes 580, Cidade Universitária, 05508-900, São Paulo, SP, Brasil
}

\begin{abstract}
RESUMO: A situação em que o Brasil e outros países em desenvolvimento se encontram hoje, de meros compradores de tecnologias importadas ou pagadores de royalties para laboratórios farmacêuticos estrangeiros, torna o processo de ampliação do sistema de saúde vigente muito oneroso ou, muitas vezes, não atende a suas necessidades específicas. O renovado interesse mundial, observado nos últimos anos, por produtos derivados da biodiversidade, tais como fitoterápicos, fitofármacos, cosméticos e suplementos alimentares, vêm estimulando investimentos de países industrializados em bioprospecção. Estas constatações devem estimular o debate, sobretudo no seio de países em desenvolvimento e detentores de rica biodiversidade e de conhecimentos tradicionais, como é o caso do Brasil, sobre a necessidade da instituição de modelos de saúdes nacionais, pautados em suas aptidões e carências, e sobre as oportunidades econômicas que o uso ético da biodiversidade apresenta.
\end{abstract}

Unitermos: Bioprospecção, fitoterápicos, fitomedicamentos, cosméticos, nutracêuticos, etnobotânica.

\begin{abstract}
Ethical use of the brazilian biodiversity: necessity and opportunity". Nowadays, Brazil and other developing countries are simple pharmaceutical technologies buyers or are only paying royalties to foreign laboratories. These facts make the public health system raise very expensive or can't put up attend to the specific necessities of these countries. The renewed global interest for natural products, such as phytotherapics, phytomedicines, cosmetics and nutraceuticals, has been stimulating industrialized countries investment in bioprospection. These evidences should stimulate the arguments, specially in developing countries, rich in natural resources and traditional knowledge, like Brazil, on the necessity of national health politics, based on their need and capacity, and on the economical opportunities that the ethical use of the biodiversity presents.
\end{abstract}

Keywords: Bioprospection, phytotherapics, phytomedicines, cosmetics, nutraceuticals, ethnobotany.

\section{INTRODUÇÃO}

A criação de modelos nacionais de saúde, pautados nas aptidões e carências de países em desenvolvimento, é tida como fundamental para tornar o acesso à saúde pública mais abrangente e de melhor qualidade (World Health Organization, 2002). A situação em que estes países se encontram hoje, de meros compradores de tecnologias importadas ou pagadores de royalties para grandes laboratórios estrangeiros, que se acentuou no Brasil após a promulgação da legislação sobre propriedade intelectual, em 1996 (Vogt, 2001; A questão..., 2001; Siani, 2003), torna o processo de inclusão no sistema de saúde vigente muito oneroso ou, muitas vezes, não atende às necessidades específicas de cada país.

Especialmente em países com rica biodiversidade e conhecimentos tradicionais abundantes, como é o caso do Brasil (o Ministério do Meio Ambiente estima que populações indígenas brasileiras dominem a aplicação medicinal de 1300 plantas brasileiras - Silveira, 2003), e com elevada incidência das chamadas "doenças negligenciadas", tais como tuberculose (UNICEF et al., 2002a), malária (UNICEF et al., 2002b), mal de chagas (UNICEF et al., 2002c), esquistossomose (UNICEF et al., 2002d), leishmaniose (UNICEF et al., 2002e) e doença do sono, este debate torna-se ainda mais relevante. Isto porque o desenvolvimento de novas drogas para o tratamento destas doenças, que afetam sobretudo populações de países em desenvolvimentos, pouco interessa à indústria farmacêutica (A questão..., 2001; Morel, 2003), pois embora estes países reúnam $80 \%$ da população mundial, correspondem a apenas $20 \%$ das vendas globais de remédios (Atas, 2003). Entre 1975 e 1999, 15 novos produtos foram desenvolvidos para o tratamento destas doenças, sendo que no mesmo período surgiram 179 novas drogas para atender portadores de doenças cardiovasculares, embora ambos os grupos representem cerca de $12 \%$ do numero total de enfermos do planeta (Atas, 2003). A própria Organização Mundial da Saúde reconhece no conhecimento tradicional sobre produtos da biodiversidade um importante instrumento no desenvolvimento de novos produtos farmacêuticos para o combate de doenças que assolam as populações dos 
Tabela 1. Exemplos de fármacos isolados de espécies vegetais

\begin{tabular}{|c|c|c|}
\hline Fármaco & Classe terapêutica & Espécie vegetal \\
\hline Ácido salicílico, salicina & Analgésico & Salix alba \\
\hline Artemisinina & Antimalárico & Artemisia annua \\
\hline Atropina & Anticolinérgico & Atropa belladona \\
\hline Capsaicina & Anestésico tópico & Capsicum spp. \\
\hline Cocaína & Anestésico local & Erythroxylum coca \\
\hline Colchina & Anti-reumático & Colchicum autumnale \\
\hline Digoxina, Digitoxina & Cardiotônicos & Digitalis purpurea, D. lanata \\
\hline Dicumarol & Anticoagulante & Melilotus officinalis \\
\hline Efedrina & Adrenérgico, broncodilatador & Ephedra sinica \\
\hline Emetina & antiamebiano & Cephaelis ipecacuanha \\
\hline Ergotamina & Bloquedor adrenérgico & Claviceps purpurea \\
\hline Escopolamina & Antiparkinsoniano & Datura spp. \\
\hline Estrofantina & Cardiotônico & Strophantus spp. \\
\hline Fisostigmina & Antiglaucomatoso & Physostigma venenosum \\
\hline Morfina, Codeína & Analgésico, antitussígeno & Papaver somniferum \\
\hline Pilocarpina & Antiglaucomatoso & Pilocarpus jaborandi \\
\hline Quinina & Antimalárico & Cinchona spp. \\
\hline Reserpina & Anti-hipertensivo & Rauwolfia spp. \\
\hline Riponinina & Anticoncepcional & Ocotea rodiei \\
\hline Taxol (paclitaxel) & Anticâncer & Taxus brevifolia \\
\hline Tubocurarina & Bloqueador neuromuscular & Chondodendron tomentosum \\
\hline Vimblastina, vincrisina & Antitumorais & Catharantus roseus \\
\hline
\end{tabular}

Fonte: Simões et al., 1999, Yunes; Calixto, 2001.

países em desenvolvimento (World Health Organization, 2002) (cálculos realizados em 1997 mostram que um terço da população mundial ainda carece de acesso a medicamentos essenciais - Organización Mundial de la Salud, 2003). A opção de conduzir pesquisas a partir da indicação de plantas utilizadas por comunidades encurta o percurso do desenvolvimento de uma nova droga, já que os pesquisadores dispõem, antes mesmo de iniciarem os estudos científicos, uma indicação de qual atividade biológica esta droga poderia apresentar (Amazônia..., 2004b).

Considerando-se, ainda, a expansão mundial que os mercados de produtos derivados de plantas (fitoterápicos, suplementos alimentares, cosméticos, repelentes de insetos, corantes, etc.) vêm conquistando, e que $25 \%$ dos fármacos empregados atualmente nos países industrializados advêm, direta ou indiretamente, de produtos naturais (Yunes; Calixto, 2001) (Tabela 1), vê-se que os países detentores de grande biodiversidade têm a oportunidade de entrar em mercados bilionários, como o farmacêutico e o de suplementos alimentares, que movimentam cerca 320 e 31 bilhões de dólares/ ano, respectivamente (Simões et al., 1999; Gruenwald; Phytopharm Consulting, 2002). O uso de remédios à base de ervas cresceu 380\% nos Estados Unidos entre 1990 e 1997, enquanto que estudo realizado com a população alemã mostra que $70 \%$ das pessoas declaram recorrer à "medicina natural" como primeira escolha no tratamento de doenças menos graves ou pequenas disfunções (World Health Organization, 2003). A Tabela 2 mostra a distribuição mundial do mercado de fitoterápicos e de suplementos alimentares, em 2001.

Não obstante, a escassa inovação tecnológica em pesquisa e exploração de produtos naturais é uma das características marcantes de países em desenvolvimento. No Brasil, as inovações têm sido de baixa ou média intensidade, sendo os fitoterápicos mais vendidos no mercado brasileiro produzidos a partir de espécies

Rev. Bras. Farmacogn Braz J. Pharmacogn. 15(2):abr/jun. 2005 
Tabela 2. Mercados regionais de suplementos vitamínicos, fitoterápicos e suplementos desportivos, em 2001. valores em US\$ bi

\begin{tabular}{lllll}
\hline Região/País & $\begin{array}{l}\text { Suplementos minerais e } \\
\text { vitamínicos }\end{array}$ & Fitoterápicos & $\begin{array}{l}\text { Suplementos } \\
\text { Desportivos }\end{array}$ & Total \\
\hline América do Norte & 7,9 & 3,9 & 4,5 & 16,3 \\
Europa & 5,5 & 6,9 & 2,5 & 14,9 \\
Ásia & 1,6 & 5,1 & 1,1 & 7,8 \\
Japão & 3,5 & 2,3 & 1,4 & 7,2 \\
América do Sul & 0,8 & 0,6 & 0,3 & 1,7 \\
Demais & 1,2 & 0,8 & 0,6 & 2,6 \\
Total & 20,6 & 19,6 & 10,4 & 50,6 \\
\hline
\end{tabular}

Fonte: Gruenwald; Phytopharm Consulting, 2002.

Tabela 3. Aquisições de pequenos laboratórios fitoterápicos por grandes empresas

\begin{tabular}{ll}
\hline Laboratório comprador & Laboratório fitoterápico adquirido \\
\hline American Home Products & Dr. Munch \\
Fugisawa & Klinge \\
J\&J -Jansen & Woelm Pharma \\
Pfizer & Mack \\
Rhone Poulenc & Nateman \\
Sanofi & Platorgan \\
Smithkline Beecham & Fink \\
Searle & Heumann \\
\hline
\end{tabular}

Fonte: Wilkinson; Wahlqvist; Clarh, 2002.

estrangeiras (Wagner, 2002). Por outro lado, grandes empresas sediadas em países industrializados, como Alemanha, França, Estados Unidos e Japão (Tabela 3), vêm aplicando competências científicas e tecnológicas no desenvolvimento de produtos derivados de plantas medicinais, muitas vezes oriundas dos países em desenvolvimento e com emprego tradicional, e se consolidando como líderes neste crescente e promissor mercado (Yunes; Pedrosa; Cechinel Filho, 2001). Cabe destacar que, na maioria das vezes, não há uma partilha de benefícios com o país de origem da matéria-prima ou com as comunidades tradicionais que lhes indicaram as aplicações das plantas convertidas em um produto final (Lôbo, 2003; Menconi; Rocha, 2003).

\section{DISCUSSÃO}

Mais recentemente, o governo brasileiro vem sinalizando que pretende reverter a situação acima exposta, por exemplo, através da recém instauração do "Fórum de Competitividade da Cadeia Farmacêutica" (pelo Ministério do Desenvolvimento, Indústria e Comércio Exterior), que dedica um capítulo à questão dos fitoterápicos (Silveira, 2003), ou por meio do Conselho Nacional de Desenvolvimento Científico e Tecnológico (CNPq) e da Fundação de Amparo à Pesquisa do Estado de São Paulo (FAPESP), que criaram programas de estímulo às parcerias entre o setor produtivo e os centros de ciência e tecnologia, como universidades e centros públicos de pesquisa (Investimento..., 2001). A própria política de 
normatização do mercado de produtos derivados de plantas medicinais, levada a cabo pelo Ministério da Saúde por meio da Agência Nacional de Vigilância Sanitária (ANVISA), a instauração fóruns de debates e de leis sobre o acesso à biodiversidade (Amazônia..., 2004a), as discussões sobre o acesso aos conhecimentos tradicionais e, notadamente, a recém aprovação do primeiro fitomedicamento brasileiro (Acheflan, um antiinflamatório elaborado a partir da espécie Cordia verbenacea - Ereno, 2005), com comprovações de eficácia e segurança, são demonstrações de que há uma preocupação governamental e de que este mercado deve se profissionalizar nos próximos anos.

Um dos fatores fundamentais para se atingir o desejado desenvolvimento biotecnológico, no setor farmacêutico, é a formulação de uma política de saúde oficial que fomente o desenvolvimento da indústria farmacêutica nacional e possibilite o acesso eqüitativo de seus cidadãos a medicamentos apropriados para o tratamento de suas enfermidades (como o fez a Austrália na década de 1990, tendo apresentado resultados positivos menos de uma década depois). A interação de empresas com universidades e centros de pesquisa é essencial, pois o governo estimularia a pesquisa e o desenvolvimento de produtos de que o país necessita, enquanto que as empresas manteriam suas atenções voltadas ao mercado, procurando novas oportunidades de negócios. Um exemplo é a recém-criada parceria entre o Centro de Texinologia Aplicada (CAT) e o consórcio de indústrias farmacêuticas constituído pelos Laboratórios BiolabSanus, União Química e Biosintética, que resultou na descoberta de um potente analgésico isolado do veneno de cascavel (Crotalus terrificus) e de 17 peptídeos, isolados do cérebro de jararaca (Bothrops jararaca), com propriedades anti-hipertensivas. Testes complementares estão em andamento, visando-se o desenvolvimento de produtos a serem lançados no mercado (Vasconcelos, 2004). Ainda neste sentido, cabe mencionar a fundação, em 2004, da Ybios, empresa resultante de uma joint venture entre Natura, Centroflora e Orsa Florestal, cuja proposta é a gestão de portfolios de projetos de pesquisa, voltada para a descoberta e o desenvolvimento de produtos originários da biodiversidade brasileira, no âmbito da saúde. A estratégia básica desta nova empresa, segundo seus diretores, é ocupar a lacuna existente entre mercado e universidade, por meio da criação de redes de cooperação, aproveitando os conhecimentos da medicina popular, as capacidades instaladas e os núcleos de conhecimentos específicos dispersos entre os institutos de pesquisa e as universidades brasileiras.

\section{CONCLUSÃO}

A prospecção ética da biodiversidade, visando agregar ciência e tecnologia a seus produtos, passa a ser de importância estratégica para os países em desenvolvimento, sendo um instrumento tanto para a descoberta de alternativas para o tratamento de doenças típicas destes países, como para estimular o crescimento de suas economias (Miguel; Miguel, 2004). Se considerarmos que o Brasil pertence a uma minoria de países ditos megadiversos (contando com aproximadamente 200.000 espécies registradas - Lewinsohn; Prado, 2002 - e com cerca de $20 \%$ de toda a flora mundial - Sant'Ana; Assad, 2002) que se distingue por seu nível de desenvolvimento em pesquisa científica, contando com universidades e institutos de pesquisa bem equipados, com pesquisadores preparados (que contribuem com aproximadamente 1,2\% da produção científica mundial - Siani, 2003) e, ainda, com comunidades tradicionais detentoras de amplos conhecimentos de espécies vegetais e animais, conclui-se que o país tem potencial para ocupar lugar de destaque, em biotecnologia, no cenário internacional.

\section{REFERÊNCIAS}

Amazônia aprova debate sobre biodiversidade. Panorama Brasil. Panaroma Brasil. Disponível em: http:// www. panoramabrasil.com.br/por/index.asp, acessada em maio de 2004a.

Amazônia contra a malária 2004b. Rev Pesq Fapesp 100: 8789.

A questão das patentes na política brasileira de fármacos 2001. Comciência 25. Disponível em: www.comciencia.br, acessada em maio 2004.

Atas L 2003. Atenção à pobreza. Rev Pesq Fapesp 91.

Ereno D 2005. Da natureza para a farmácia. Rev Pesq Fapesp 110: 78-81.

Gruenwald J, Phytopharm Consulting 2002. The North American, European and Asian phytomedicine and natural products markets. Fórum de Debates - Fitomedicamentos e Produtos Naturais. São Paulo.

Investimento em pesquisa de fármacos no Brasil ainda é pequena 2001. Comciência 25. Disponível em: http:// www.comciencia.br/reportagens/farmacos/farma11. htm, acessada em maio 2004.

Lewinsohn TM, Prado PI 2002. Biodiversidade brasileira: síntese do estado atual do conhecimento. São Paulo: Contexto.

Lôbo I 2003. Biopirataria dá prejuízo de US\$ 1 bilhão por ano ao Brasil. Agência Brasil. Disponível em: http://www. radiobras.gov.br, acessada em setembro de 2003.

Menconi D, Rocha L 2003. Riqueza ameaçada. Isto É 1773: 92-98.

Miguel MD, Miguel OG 2004. Desenvolvimento de fitoterápicos. Ribeirão Preto: Tecmedd.

Morel CM 2003. Neglected diseases: under-funded research and inadequate health interventios. Eur Mol Biol Org Reports 4: S35-S38.

Organización Mundial de la Salud 2003. Perspectivas políticas de la OMS sobre medicamentos. Ginebra: Organización Mundial de la Salud.

Sant'Ana PJP, Assad AL 2002. O contexto brasileiro para a bioprospecção. Biotecnologia ciência $e$ desenvolvimento 29 2002. Disponível em: http:// www.biotecnologia.com.br, acessada em dezembro de 2004.

Siani AC 2003. Desenvolvimento tecnológico de fitoterápicos -

Rev. Bras. Farmacogn.

Braz J. Pharmacogn.

15(2):abr/jun. 2005 
plataforma metodológica. Rio de Janeiro: Scriptorio Comunicação.

Silveira JH 2003. Uso da biodiversidade para produção de medicamentos. Gazeta Mercantil, São Paulo, 25 jun.

Simões CMO, Schenkel EP, Gosmann G, Mello JCP, Mentz LA, Petrovick PR 1999. Farmacognosia: - da planta ao medicamento. Porto Alegre: UFRGS; Florianópolis: UFSC.

UNICEF, UNDP, WORLD BANK, WHO 2002a. Special Programme for Research and Training in Tropical Deseases. Strategic Direction for research: Tuberculosis. Disponível em: http://www.who.int/ tdr/diseases/tb/files/direction.pdf, acessado em jun. 2004.

UNICEF, UNDP, WORLD BANK, WHO 2002b. Special Programme for Research and Training in Tropical Deseases. Strategic Direction for research: Malaria. Disponível em: http://www.who.int/tdr/diseases/ malaria/files/direction.pdf, acessada em jun. 2004.

UNICEF, UNDP, WORLD BANK, WHO 2002c. Special Programme for Research and Training in Tropical Deseases. Strategic Direction for research: Chagas disease. Disponível em: http://www.who.int/tdr/ diseases/chagas/files/direction.pdf, acessada em jun. 2004.

UNICEF, UNDP, WORLD BANK, WHO 2002d. Special Programme for Research and Training in Tropical Deseases. Strategic Direction for research: Schistosomiasis. Disponível em: http://www.who. int/tdr/diseases/schisto/files/direction.pdf, acessada em jun. 2004.

UNICEF, UNDP, WORLD BANK, WHO 2002e. Special Programme for Research and Training in Tropical Deseases. Strategic Direction for research: Leishmaniasis. Disponível em: http://www.who.int/ $\mathrm{tdr} /$ diseases/leish/files/direction.pdf, acessada em jun. 2004.

Vasconcelos Y 2004. Fábrica de moléculas. Rev Pesq Fapesp 100: 134-137.

Vogt C 2001. Fármacos e medicamentos: urgências. Comciência 25. Disponível em: http://www.comciencia.br, acessada em maio 2004.

Wagner JDA 2002. A importância dos produtos de origem natural no atendimento à saúde. Fórum de Debates - Fitomedicamentos e Produtos Naturais. São Paulo, Brasil.

Wilkinson JA, Wahlqvist ML, Clarh J 2002. New food and pharmaceutical products from agriculture. A report for the Rural Industries Research and Development Corporation. Kingston: RIRDC Publication.

World Health Organization 2002. WHO police perspectives on medicines. Geneva: World Heath Organization.

World Health Organization 2003. Draft WHO guidelines on safety monitoring and pharmacovigilance of herbal medicines. Amsterdan: World Health Organization.

Yunes RA, Pedrosa RC, Cechinel Filho V 2001. Fármacos e fitoterápicos: a necessidade do desenvolvimento da indústria de fitoterápicos e fitofármacos no Brasil. Quím. Nova 24: 147-152.

Yunes RA, Calixto JB 2001. Plantas medicinais - sob a ótica da química medicinal moderna. Chapecó: Argos. 